\title{
Unified formulation for inhomogeneity-driven instabilities in the lower-hybrid range
}

\author{
O. J. G. Silveira* and L. F. Ziebell ${ }^{\dagger}$ \\ Instituto de Física, Universidade Federal do Rio Grande do Sul (UFRGS), Caixa Postal 15051, 91501-970 Porto Alegre, \\ RS, Brazil \\ R. Gaelzer \\ Instituto de Física e Matemática, Universidade Federal de Pelotas (UFPel), Caixa Postal 354, 96010-900 Pelotas, \\ RS, Brazil \\ Peter H. Yoon ${ }^{\S}$ \\ Institute for Physical Science and Technology, University of Maryland, College Park, Maryland 20742
}

(Received 6 September 2001; published 19 February 2002)

\begin{abstract}
A local dispersion relation that describes inhomogeneity-driven instabilities in the lower-hybrid range is derived following a procedure that correctly describes energy exchange between waves and particles in inhomogeneous media, correcting some inherent ambiguities associated with the standard formalism found in the literature. Numerical solutions of this improved dispersion relation show that it constitutes a unified formulation for the instabilities in the lower-hybrid range, describing the so-called modified two-stream instability, excited by the ion cross-field drift, including the ion Weibel instability, and also describing the lower-hybrid drift instability, which is due to inhomogeneity effects on the electron population.
\end{abstract}

DOI: 10.1103/PhysRevE.65.036407

PACS number(s): 52.25.Mq, 52.35.-g, 94.30.Tz

\section{INTRODUCTION}

The plasma of earth's magnetosphere offers plenty of possibilities for the excitation of waves that may feed on the free energy available due to inhomogeneities. In the magnetotail, drift instabilities may play an important role as the energy dissipation mechanism. The electron density, magnetic field, and temperature gradients are known to drive the so-called lower-hybrid drift instability (LHDI) [1-7], while the crossfield drift of the unmagnetized ions drives the modified twostream instability (MTSI) [8-12]. In general, both instabilities may be simultaneously present in the magnetotail.

The standard formalism found in the literature usually employs a local approximation to discuss these instabilities, treating the inhomogeneity as adiabatic. However, such an approximation contains some inherent ambiguities. A relatively detailed description of these ambiguities can be found in a paper by Yoon, Lui, and Chang 1994, where an unsuccessful attempt toward a unified description of instabilities in the lower-hybrid range has been made [13]. In Ref. [13] the strongly electrostatic nature of the LHDI has been taken into account by using Poisson's equation and the relationship between charge density and electric field to obtain some of the components of the dielectric tensor, taking into account inhomogeneity effects, while Ampere's law and the relationship between current density and the electric field was used for other components. This hybrid formulation has been able to describe the LHDI, but could not be used to describe the MTSI in the limit for parallel propagation. On the other

\footnotetext{
*Email address: omar@if.ufrgs.br

${ }^{\dagger}$ Email address: ziebell@if.ufrgs.br

\#Email address: rudi@ufpel.tche.br

${ }^{\S}$ Email address: yoonp@ipst.umd.edu
}

hand, the usual dispersion relation derived with use of the relationship between current density and electric field could describe the MTSI/IWI (ion Weibel instability), but failed to obtain the LHDI [13].

Taking into account this situation, and aiming to formulate a unified and consistent local theory for these inhomogeneity-driven phenomena, we have recently derived a rather general expression for the electron linear dielectric response function, which can be readily utilized in the stability analysis of weakly inhomogeneous media [14]. Such a result is a culmination of a series of earlier efforts in which inhomogeneous plasma parameters [15-20] and inhomogeneous magnetic field [21-23] have been discussed. The derivation of the general expression is based on an iterative method termed "the BGI procedure," after Beskin, Gurevich, and Istomin who pioneered such a method. This procedure was developed in order to ensure that the absorption coefficient obtained from the solution of the dispersion relation is related to an effective energy exchange between waves and particles [24], which the adiabatic local theory does not.

In the present paper we derive a proper dispersion relation, taking into account the relevant inhomogeneity effects, and use with this dispersion relation the components of the dielectric tensor obtained with the general formalism described in Ref. [14], particularized to the case of drift instabilities in the lower-hybrid frequency range, for propagation in the plane perpendicular to the direction of the inhomogeneity gradients. It is found that the resulting dispersion relation offers an unified description of both the LHDI and the MTSI, and also describes the so-called IWI, a purely growing instability that appears for purely parallel propagation. Such unified description was not yet available in the literature, to the best of our knowledge.

The structure of the paper is the following. In Sec. II we 


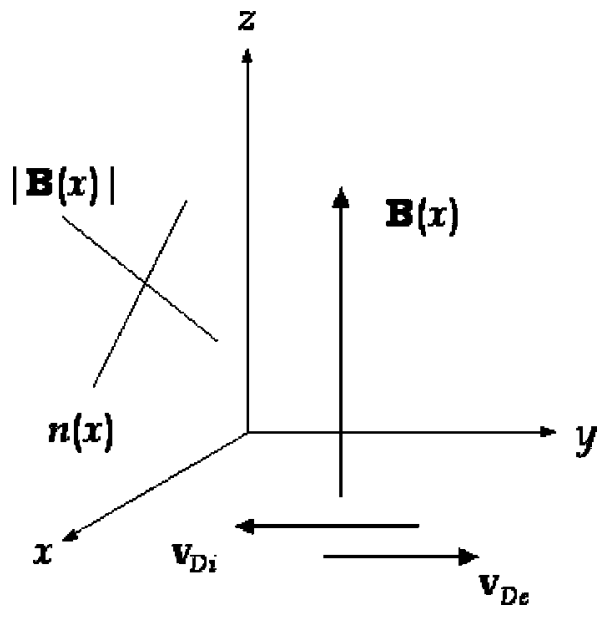

FIG. 1. Schematic representation of the inhomogeneous profiles of magnetic field and density in the slab geometry, indicating the orientation of the electron and ion drift velocities.

describe the model of the inhomogeneous region and write down the final expressions for the components of the dielectric tensor to be used in the dispersion relation. In Sec. III we establish the appropriate dispersion relation that incorporates the relevant inhomogeneity effects. In Sec. IV we conduct a series of numerical analyses of the dispersion relation, considering parameters relevant to the case of earth's magnetotail. Finally, some comments on the results obtained appear in Sec. V.

\section{MODEL OF THE INHOMOGENEOUS REGION AND THE COMPONENTS OF THE DIELECTRIC TENSOR}

Let us consider a slab of magnetized plasma, with magnetic field pointing along the $z$ direction, featuring inhomogeneities in magnetic field and density, along the $x$ direction, with the following profiles:

$$
\begin{gathered}
\mathbf{B}(x)=B_{0}\left(1+\epsilon_{B} x\right) \mathbf{e}_{z}, \\
n(x)=n_{0}\left(1-\epsilon_{n} x\right),
\end{gathered}
$$

where $n(x)$ represents the equilibrium electron density at position $x$, assumed to be equal to the ion density, and where $\epsilon_{B}=1 / L_{B}$ and $\epsilon_{n}=1 / L_{n}$ are inverse scale lengths that characterize the inhomogeneities in magnetic field and density, respectively. These profiles may represent, for instance, the magnetic field and density at the magnetotail of the earth. Figure 1 is a schematic view of the slab geometry, featuring the gradients of magnetic field and density that are perpendicular to the ambient magnetic field, and the ion and electron diamagnetic drift velocities, which occur due to the density inhomogeneity.

In an inhomogeneous slab as we are considering the electron distribution function depends on $\boldsymbol{v}$ and $\mathcal{X}_{e}=x$ $-v_{y} /\left|\Omega_{e}\right|$, the space dependent constant of motion that represents the $x$ component of the guiding center position of the electron cyclotron orbits. If the scale length of the density inhomogeneity is much larger than the electron Larmor ra- dius $\left(L_{n} \gg r_{L e}\right.$, where $\left.r_{L e}=v_{e} /\left|\Omega_{e}\right|\right)$, the electrons distribution can be given by the following approximated form

$$
\begin{aligned}
F_{e}\left(\boldsymbol{v}, \mathcal{X}_{e}\right) & \simeq f_{e}(x, \boldsymbol{v})-\frac{v_{y}}{\left|\Omega_{e}\right|} \frac{\partial f_{e}}{\partial x}(x, \boldsymbol{v}) \\
& =\left(1+\sqrt{2} \frac{v_{D e} v_{y}}{v_{e}^{2}}\right) f_{e}(x, \boldsymbol{v}), \\
f_{e}(x, \boldsymbol{v}) & =\frac{n(x)}{(2 \pi)^{3 / 2} v_{e}^{3}} \exp \left(-\frac{v^{2}}{2 v_{e}^{2}}\right),
\end{aligned}
$$

where $v_{e}=\sqrt{T_{e} / m_{e}}$ is the electron thermal speed, $T_{e}$ being the electron temperature, and $v_{D e}=\epsilon_{n} v_{e}^{2} / \sqrt{2}\left|\Omega_{e}\right|$ is the electron diamagnetic drift speed.

The ions can be considered as unmagnetized for waves characterized by the inequalities, $\omega \gg \Omega_{i}$ and $r_{L i} \gg \lambda$, where $\omega$ is the wave angular frequency, $\Omega_{i}$ is the ion cyclotron angular frequency, $r_{L i}$ is the ion Larmor radius, and $\lambda$ is the wavelength. Therefore, it is not necessary for us to use for the ions the expression developed in Ref. [14], but instead we may simply treat the ion distribution as a drifting Maxwellian,

$$
f_{i}=\frac{n(x)}{(2 \pi)^{3 / 2} v_{i}^{3}} \exp \left(-\frac{v_{x}^{2}+\left(v_{y}+v_{D i}\right)^{2}+v_{z}^{2}}{2 v_{i}^{2}}\right),
$$

where $v_{i}=\sqrt{T_{i} / m_{i}}$ is the ion thermal speed and $v_{D i}$ $=\epsilon_{n} v_{i}^{2} /\left(\sqrt{2} \Omega_{i}\right)$ is the ion (diamagnetic) cross-field drift speed. This distribution function for the unmagnetized ions allows for simplicity in the description of the ion contribution for the dispersion relation, while featuring the cross-field drift movement that is the driving mechanism for the MTSI instability.

We now consider the case of propagation in the $y z$ plane, which is the plane perpendicular to the direction of the inhomogeneity gradients, and frequencies in the vicinity of the lower-hybrid frequency, which means that the only important contributions are those that pertain to the harmonics $n=0$ and $n= \pm 1$ in the electron response functions.

The dielectric tensor is given by

$$
\overleftrightarrow{\varepsilon}=\overleftrightarrow{I}+\overleftrightarrow{\chi}^{e}+\overleftrightarrow{\chi}^{i},
$$

where $\overleftrightarrow{\chi}^{e}$ corresponds to the electron dielectric susceptibility tensor and $\overleftrightarrow{\chi}^{i}$ corresponds to the same for the ions. The components of $\overleftrightarrow{\chi}^{e}$ and $\vec{\chi}^{i}$, given below (details of the derivation appears in Appendix A), include the corrections, which follow from the above-mentioned BGI procedure, to the standard expressions found in the literature (see, e.g., [13]),

$$
\begin{aligned}
\chi_{x x}^{e}= & \frac{1}{\bar{\omega}^{2}} \frac{\omega_{p e}^{2}}{\Omega_{e}^{2}} \frac{m_{i}}{m_{e}}\left(-\sqrt{2} \frac{q_{n}}{q_{\|}} \nu_{e}^{3} \mathcal{H}_{0}^{\prime}\left(\nu_{e}^{2}\right) Z\left(z_{0}\right)\right. \\
& \left.+4 z_{0} \int_{0}^{\infty} d u u^{3} \mathbf{e}^{-u^{2}} J_{1}^{2}\left(\sqrt{2} \nu_{e} u\right) Z\left(\bar{z}_{0}\right)\right),
\end{aligned}
$$




$$
\begin{aligned}
& \chi_{y y}^{e}=\frac{1}{\bar{\omega}^{2}} \frac{\omega_{p e}^{2}}{\Omega_{e}^{2}} \frac{m_{i}}{m_{e}}\left[\frac { \sqrt { 2 } } { 2 } \frac { q _ { n } } { q _ { \| } } \frac { 1 } { \nu _ { e } } \sum _ { n = \pm 1 } \left(\mathcal{H}_{1}\left(\nu_{e}^{2}\right)\right.\right. \\
& \left.+\frac{n \bar{\omega}}{\left(m_{i} / m_{e}\right)^{1 / 2}}\left[\mathcal{H}_{1}\left(\nu_{e}^{2}\right)-\mathcal{H}_{2}\left(\nu_{e}^{2}\right)\right]\right) Z\left(z_{n}\right) \\
& +\frac{2 z_{0}}{\nu_{e}^{2}}\left(\int_{0}^{\infty} d u u \mathbf{e}^{-u^{2}} J_{1}^{2}\left(\sqrt{2} \nu_{e} u\right) \sum_{n= \pm 1} Z\left(\bar{z}_{n}\right)\right. \\
& -\frac{q_{B}}{q_{\|}} \sum_{n= \pm 1} Z^{\prime}\left(z_{n}\right) \int_{0}^{\infty} d u u^{2} \mathbf{e}^{-u^{2}} \\
& \left.\times J_{1}\left(\sqrt{2} \nu_{e} u\right) J_{2}\left(\sqrt{2} \nu_{e} u\right)\right) \text {, } \\
& \chi_{z z}^{e}=\frac{1}{\bar{\omega}^{2}} \frac{\omega_{p e}^{2}}{\Omega_{e}^{2}} \frac{m_{i}}{m_{e}}\left(-\frac{\sqrt{2}}{2} \frac{q_{n}}{q_{\|}} \nu_{e} z_{0} \mathcal{H}_{0}\left(\nu_{e}^{2}\right) Z^{\prime}\left(z_{0}\right)\right. \\
& \left.-2 z_{0} \int_{0}^{\infty} d u u \mathbf{e}^{-u^{2}} J_{0}^{2}\left(\sqrt{2} \nu_{e} u\right) \bar{z}_{0} Z^{\prime}\left(\bar{z}_{0}\right)\right), \\
& \chi_{x y}^{e}=-\chi_{y x}^{e}=\frac{i}{\bar{\omega}^{2}} \frac{\omega_{p e}^{2}}{\Omega_{e}^{2}} \frac{m_{i}}{m_{e}}\left[-\frac{q_{n}}{q_{\perp}} \nu_{e}^{2} z_{0} \mathcal{H}_{0}^{\prime}\left(\nu_{e}^{2}\right) Z\left(z_{0}\right)\right. \\
& +\frac{2 \sqrt{2} z_{0}}{\nu_{e}}\left(\int_{0}^{\infty} d u u^{2} \mathbf{e}^{-u^{2}} J_{1}\left(\sqrt{2} \nu_{e} u\right) J_{1}^{\prime}\left(\sqrt{2} \nu_{e} u\right)\right. \\
& \times \sum_{n= \pm 1} n Z\left(\bar{z}_{n}\right)+\frac{1}{2} \frac{q_{B}}{q_{\|}} \sum_{n= \pm 1} n Z^{\prime}\left(z_{n}\right) \\
& \left.\left.\times \int_{0}^{\infty} d u u^{3} \mathbf{e}^{-u^{2}} J_{2}^{2}\left(\sqrt{2} \nu_{e} u\right)\right)\right] \text {, } \\
& \chi_{x z}^{e}=-\chi_{z x}^{e}=-\frac{i}{\bar{\omega}^{2}} \frac{\omega_{p e}^{2}}{\Omega_{e}^{2}} \frac{m_{i}}{m_{e}}\left(\frac{1}{2} \frac{q_{n}}{q_{\|}} \nu_{e}^{2} \mathcal{H}_{0}^{\prime}\left(\nu_{e}^{2}\right) Z^{\prime}\left(z_{0}\right)\right. \\
& \left.+2 z_{0} \int_{0}^{\infty} d u u^{2} \mathbf{e}^{-u^{2}} J_{0}\left(\sqrt{2} \nu_{e} u\right) J_{1}\left(\sqrt{2} \nu_{e} u\right) Z^{\prime}\left(\bar{z}_{0}\right)\right), \\
& \chi_{y z}^{e}=\chi_{z y}^{e}=\frac{1}{\bar{\omega}^{2}} \frac{\omega_{p e}^{2}}{\Omega_{e}^{2}} \frac{m_{i}}{m_{e}}\left[-\frac{\sqrt{2}}{4} \frac{q_{n}}{q_{\perp}} \nu_{e} z_{0} \mathcal{H}_{0}^{\prime}\left(\nu_{e}^{2}\right) Z^{\prime}\left(z_{0}\right)\right. \\
& +\frac{\sqrt{2} z_{0}}{\nu_{e}}\left(\int_{0}^{\infty} d u u \mathbf{e}^{-u^{2}} J_{1}^{2}\left(\sqrt{2} \nu_{e} u\right) \sum_{n= \pm 1} n Z^{\prime}\left(\bar{z}_{n}\right)\right. \\
& +\frac{\sqrt{2}}{2} \frac{q_{B}}{q_{\|}} \frac{1}{\nu_{e}} \sum_{n= \pm 1} n\left[z_{n} Z^{\prime}\left(z_{n}\right)+Z\left(z_{n}\right)\right] \\
& \times \int_{0}^{\infty} d u u \mathbf{e}^{-u^{2}}\left[J_{1}^{2}\left(\sqrt{2} \nu_{e} u\right)\right. \\
& \left.\left.\left.+\sqrt{2} \nu_{e} u J_{1}\left(\sqrt{2} \nu_{e} u\right) J_{2}\left(\sqrt{2} \nu_{e} u\right)\right]\right)\right],
\end{aligned}
$$

for the electrons, and

$$
\begin{gathered}
\chi_{x x}^{i}=\frac{1}{\bar{\omega}^{2}} \frac{\omega_{p e}^{2}}{\Omega_{e}^{2}} z_{i} Z\left(z_{i}\right) \\
\chi_{y y}^{i}=\frac{1}{\bar{\omega}^{2}} \frac{\omega_{p e}^{2}}{\Omega_{e}^{2}}\left[\frac{q_{\|}^{2}}{q^{2}} z_{i} Z\left(z_{i}\right)-\left(\frac{q_{\perp}}{q} z_{i}-\frac{v_{D i}}{v_{i}}\right)^{2} Z^{\prime}\left(z_{i}\right)\right] \\
\chi_{z z}^{i}=\frac{1}{\bar{\omega}^{2}} \frac{\omega_{p e}^{2}}{\Omega_{e}^{2}} z_{i}\left(\frac{q_{\perp}^{2}}{q^{2}} Z\left(z_{i}\right)-\frac{q_{\|}^{2}}{q^{2}} z_{i} Z^{\prime}\left(z_{i}\right)\right) \\
\chi_{y z}^{i}=\chi_{z y}^{i}=-\frac{1}{\bar{\omega}^{2}} \frac{\omega_{p e}^{2}}{\Omega_{e}^{2}} \frac{q_{\|}}{q} z_{i}\left[\frac{q_{\perp}}{q} Z\left(z_{i}\right)+\left(\frac{q_{\perp}}{q} z_{i}-\frac{v_{D i}}{v_{i}}\right) Z^{\prime}\left(z_{i}\right)\right]
\end{gathered}
$$

for the ions, where $\bar{\omega}=\omega / \omega_{l h}, \omega_{l h}=\left(\Omega_{i}\left|\Omega_{e}\right|\right)^{1 / 2}$ being the lower-hybrid angular frequency, $\Omega_{i}=e B_{0} / m_{i} c$ and $\Omega_{e}=$ $-e B_{0} / m_{e} c$ being the ion and electron cyclotron frequency, respectively; $\omega_{p e}^{2}=4 \pi n_{0} e^{2} / m_{e}$ and $\omega_{p i}^{2}=4 \pi n_{0} e^{2} / m_{i}$ are the electron and ion plasma frequencies, respectively:

$$
q_{n}=\frac{c}{\omega_{p e}} \epsilon_{n}, \quad q_{B}=\frac{c}{\omega_{p e}} \epsilon_{B}, \quad q_{\perp}=\frac{c k_{\perp}}{\omega_{p e}}, \quad q_{\|}=\frac{c k_{\|}}{\omega_{p e}},
$$

$k_{\perp}$ and $k_{\|}$being the parallel and perpendicular components of the wave vector with respect to the ambient magnetic field, respectively; $\mathcal{H}_{n}(x)=I_{n}(x) \exp (-x), I_{n}(x)$ being the modified Bessel function of the first kind of order $n$, the prime indicating the derivative with respect to the argument;

$$
\begin{gathered}
\nu_{e}^{2}=\frac{k_{\perp}^{2} v_{e}^{2}}{\Omega_{e}^{2}}, \quad z_{0}=\frac{\omega}{\sqrt{2} k_{\|} v_{e}}, \\
z_{(n= \pm 1)} \equiv z_{ \pm}=\frac{\omega \mp \Omega_{e}}{\sqrt{2} k_{\|} v_{e}}=\frac{\omega \pm\left|\Omega_{e}\right|}{\sqrt{2} k_{\|} v_{e}}, \\
\bar{z}_{n}=\frac{\omega-n \Omega_{e}-k_{\perp} \epsilon_{B} v_{e}^{2} u^{2} / \Omega_{e}}{\sqrt{2} k_{\|} v_{e}}, \quad z_{i}=\frac{\omega+\sqrt{2} k_{\perp} v_{D i}}{\sqrt{2} k v_{i}} .
\end{gathered}
$$

$Z(z)$ and $Z^{\prime}(z)$ are the familiar plasma dispersion (FriedConte) function and its derivative, respectively.

One of the important consequences of the "BGI" correction is that the above dielectric tensor elements preserve the correct symmetry with respect to the off-diagonal elements, namely,

$$
\epsilon_{x y}=-\epsilon_{y x}, \quad \epsilon_{x z}=-\epsilon_{z x}, \quad \epsilon_{y z}=\epsilon_{z y} .
$$

Reference [13] makes note of the breakdown of this symmetry (i.e., the Onsager symmetry) in the traditional local approximation of weakly inhomogeneous plasma linear stability problem, and attempts to correct this problem by employing components $\epsilon_{y j}(j=x, y, z)$ calculated with the use of an approximated form of the perturbed Poisson's 
equation. The resulting expressions for the tensor elements did restore the required symmetry, but it was a fortuitous happenstance rather than a result based upon first principles. The authors of Ref. [13] speculated whether the breakdown of Onsager symmetry was the result of approximated orbit calculation associated with the electrons. However, in reality the problem has nothing to do with the orbits, but rather it is an inherent consequence of the local approximation, which in general does not satisfy the proper energy exchange relation between the particles and waves. The BGI procedure naturally corrects this deficiency and the Onsager symmetry relation results as a corollary, as Eq. (3) clearly demonstrates. In short, we believe that Eq. (3) represents a more systematic and accurate formula appropriate for local linear stability analysis of weakly inhomogeneous plasmas in the lowerhybrid frequency range.

At this point, a brief overview of the BGI procedure may be appropriate. As explained elsewhere [18,22], the procedure first involves the computation of the customary expression of the local dielectric tensor on the basis of the relationship between current density and electric field, but incorporating inhomogeneity effects. This customary expression does not satisfy Onsager symmetry. A set of remedial prescriptions generally outlined in Ref. [24] is then applied to this preliminary form. It is important to point out that this procedure is not ad hoc, but is based upon firm theoretical ground. The result is Eq. (3). The specific details have been worked out elsewhere $[15,22,24]$. We emphazise that in Eq. (3), only the resonant particles contribute to the antiHermitian part of the susceptibility tensor, thereby describing correctly the energy exchange between waves and particles. If Landau damping is absent, the components of the susceptibility tensor are Hermitian.

\section{THE DISPERSION RELATION}

In order to obtain the dispersion relation appropriated to deal with instabilities in a weakly inhomogeneous plasma, in which we will utilize the components of the susceptibility tensor that incorporate inhomogeneity effects, as given by Eq. (3), we start from Maxwell's equations, take the curl of Ampère's law, use Faraday's law, and obtain

$$
-\frac{1}{c^{2}} \frac{\partial^{2} \mathbf{E}}{\partial t^{2}}-\boldsymbol{\nabla}(\boldsymbol{\nabla} \cdot \mathbf{E})+\nabla^{2} \mathbf{E}=\frac{4 \pi}{c^{2}} \frac{\partial \mathbf{J}}{\partial t} .
$$

Using Gauss's law of electricity, this equation becomes the following:

$$
-\frac{1}{c^{2}} \frac{\partial^{2} \mathbf{E}}{\partial t^{2}}+\nabla^{2} \mathbf{E}=4 \pi \nabla \rho+\frac{4 \pi}{c^{2}} \frac{\partial \mathbf{J}}{\partial t}
$$

Considering now the WKB approximation for $\mathbf{E}, \mathbf{J}$, and $\rho$, Eq. (6) can be transformed into an algebraic equation

$$
\frac{\omega^{2}}{c^{2}} \mathbf{E}-k^{2} \mathbf{E}=i 4 \pi k \rho-i \frac{4 \pi \omega}{c^{2}} \mathbf{J},
$$

with the current density being related to the wave electric field by a constitutive relation,

$$
\mathbf{J}=-i \frac{\omega}{4 \pi} \overleftrightarrow{\chi} \cdot \mathbf{E},
$$

where for weakly inhomogeneous media the tensor $(\vec{\chi})$ is the effective susceptibility tensor. For the geometry utilized in the present paper and for frequencies in the lower-hybrid frequency range, the components of the effective susceptibility tensor are given by Eq. (3). Regarding the charge density $\rho$, it is also related to the wave electric field by a constitutive relation. However, instead of following steps similar to those utilized to obtain the constitutive relation between $\mathbf{J}$ and $\mathbf{E}$, for $\rho$ we adopt a shortcut and use the continuity equation,

$$
\rho(\boldsymbol{r}, \omega)=-\frac{i}{\omega} \boldsymbol{\nabla} \cdot \mathbf{J}(\mathbf{r}, \omega),
$$

obtaining, after use of Eq. (8),

$\rho(\mathbf{r}, \omega)=-\frac{1}{4 \pi} \boldsymbol{\nabla} \cdot[\overleftrightarrow{\chi}(\boldsymbol{k}, \boldsymbol{r}, \omega) \cdot \mathbf{E}] \approx-\frac{1}{4 \pi}[i \boldsymbol{k} \cdot \overleftrightarrow{\chi}+\boldsymbol{\nabla} \cdot \overleftrightarrow{\chi}] \cdot \boldsymbol{E}$.

Notice that this expression for the charge density contains space derivatives of $(\vec{\chi})$, which are of the same order as gradient terms taken into account in the derivation of the susceptibility for inhomogeneous plasmas, and which are therefore to be maintained in the wave equation.

Inserting expressions (8) and (10) into Eq. (7), the outcome is a set of algebraic equations for the wave electric field,

$$
\frac{\omega^{2}}{c^{2}} \mathbf{E}-k^{2} \mathbf{E}=\boldsymbol{k}[\boldsymbol{k} \cdot \overleftrightarrow{\chi}-i(\boldsymbol{\nabla} \cdot \overleftrightarrow{\chi})] \cdot \mathbf{E}-\frac{\omega^{2}}{c^{2}} \overleftrightarrow{\chi} \cdot \mathbf{E},
$$

whose solubility condition is the following dispersion relation:

$$
\operatorname{det}\left[\left(1-N^{2}\right) \delta_{i j}+\chi_{i j}-N_{i} N_{l} \chi_{l j}+i N_{i} \frac{c}{\omega} \frac{\partial \chi_{l j}}{\partial x_{l}}\right]=0,
$$

where Einstein's sum rule for repeated indexes has been used and where $N=c k / \omega$.

For the situation discussed in the present paper the only nonvanishing space derivative of the susceptibility is the $x$ derivative, which can be written as follows:

$$
\frac{\partial \chi_{i j}}{\partial x}=-\epsilon_{B} \eta_{i j},
$$

where

$$
\eta_{x x}=0
$$




$$
\begin{aligned}
\eta_{y y}= & -\frac{1}{\bar{\omega}}\left(\frac{2}{\beta_{e}}\right)^{2} \frac{\omega_{p e}^{2}}{\Omega_{e}^{2}}\left(\frac{m_{i}}{m_{e}}\right)^{1 / 2} \frac{1}{q_{\|}^{2} q_{\perp}^{2}} \\
& \times \sum_{n= \pm 1} n \int_{0}^{\infty} d u u \mathbf{e}^{-u^{2}} Z^{(1)}\left(\bar{z}_{n}\right) J_{1}^{2}\left(\sqrt{2} \nu_{e} u\right), \\
& \eta_{z z}=0, \\
& \left.\times \sum_{n= \pm 1}^{x y}\right)= \pm i \frac{1}{\bar{\omega}}\left(\frac{2}{\beta_{e}}\right)^{3 / 2} \frac{\omega_{p e}^{2}}{\Omega_{e}^{2}}\left(\frac{m_{i}}{m_{e}}\right)^{1 / 2} \frac{\sqrt{2}}{q_{\|}^{2} q_{\perp}} \\
& \times J_{1}\left(\sqrt{2} \nu_{e} u\right) J_{1}^{\prime}\left(\sqrt{2} \nu_{e} u\right), \\
\eta\left(\begin{array}{c}
y z \\
z y
\end{array}\right)= & \frac{1}{\bar{\omega}}\left(\frac{2}{\beta_{e}}\right)^{3 / 2} \frac{\omega_{p e}^{2}}{\Omega_{e}^{2}}\left(\frac{m_{i}}{m_{e}}\right)^{1 / 2} \frac{1}{\sqrt{2} q_{n}^{2} q_{\perp}} \\
& \times \sum_{n= \pm 1} n^{2} \int_{0}^{\infty} d u u \mathbf{e}^{-u^{2}} Z^{(2)}\left(\overline{z_{n}}\right) J_{1}^{2}\left(\sqrt{2} \nu_{e} u\right) .
\end{aligned}
$$

The derivation of Eqs. (13) and (14) can be found in Appendix B. Using Eq. (13) and defining $N_{B}=c \epsilon_{B} / \omega$, the dispersion relation becomes

$$
\operatorname{det}\left[\left(1-N^{2}\right) \delta_{i j}+\chi_{i j}-N_{i} N_{l} \chi_{l j}-i N_{i} N_{B} \eta_{x j}\right]=0 .
$$

We remark here that the presence of the effective susceptibility in Eq. (15) assures that the process of energy exchange between wave and particles is correctly described, since its correct symmetry guarantees that the anti-Hermitian part of the dielectric tensor, responsible for wave absorption or amplification, is only due to resonant terms [14-16,21-23].

\section{NUMERICAL ANALYSIS OF THE DISPERSION RELATION}

We now present sample examples of the full numerical solution of the dispersion relation given by Eq. (15), where the $\chi_{i j}$ components are given by Eqs. (3) and (4), $N_{\perp}$ $=c k_{\perp} / \omega, N_{\|}=c k_{\|} / \omega$, and $N=c k / \omega$. The above equation is solved for the normalized complex frequency $\bar{\omega}$ as a function of $q_{\|}$and $q_{\perp}$, with other quantities, $\beta_{e}, \beta_{i}, \omega_{p e} /\left|\Omega_{e}\right|$, and $v_{D i} / v_{i}$, as input parameters.

In what follows, we consider $\omega_{p e} /\left|\Omega_{e}\right|=10, v_{D i} / v_{i}=1$, $\beta_{i}=5$, and consider the idealized case of $\beta_{e}=0$. We have deliberately made the choice of these parameters that are identical to those considered in Ref. [13], so that a direct comparison can be made.

The growth rate of the unstable mode with frequency in the lower-hybrid range is shown in Fig. 2, in surface mesh plot and contour plot versus $q_{\|}$and $q_{\perp}$. Figure 2 shows many features that are similar to those appearing in Fig. 3 of

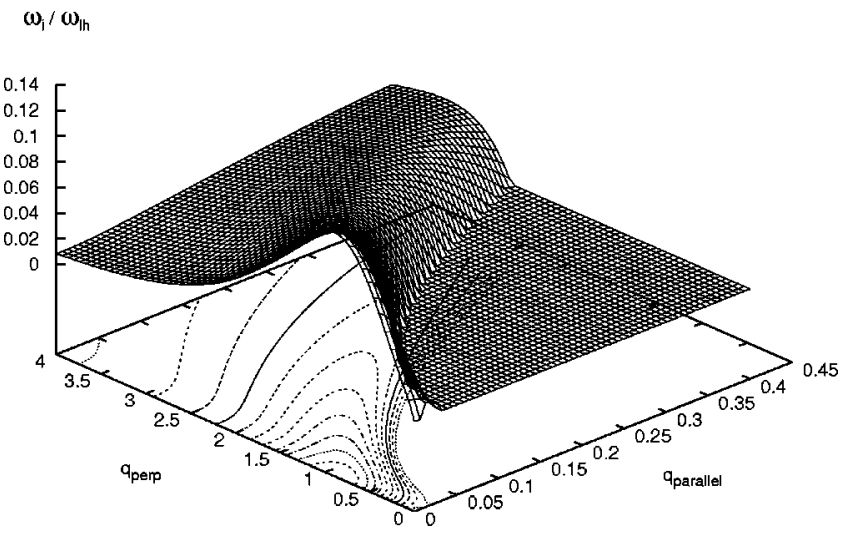

FIG. 2. Dimensionless growth rate $\bar{\omega}_{i}$ as a function of the dimensionless quantities $q_{\|}$and $q_{\perp}$ for $\omega_{p e} /\left|\Omega_{e}\right|=10, v_{D i} / v_{i}=1.0$, $\beta_{i}=5$, and $\beta_{e}=0$, fully taking into account the electron inhomogeneity.

Ref. [13], namely, the occurrence of a pronounced instability at $q_{\|}=0$, coupled to an extended unstable region for a large interval of values of finite $q_{\|}$and $q_{\perp}$. However, it is also seen the occurrence of an instability for $q_{\perp} \rightarrow 0$, which was not obtained from the formulation appearing in Ref. [13]. This is a purely growing instability, with vanishing real frequency, as it is seen in Fig. 3, which shows the real part of the frequency corresponding to the growth rates appearing in Fig. 2.

In order to investigate the nature of the instabilities obtained, we show in Fig. 4 the growth rates evaluated for a situation in which we keep the ion drift velocity, $v_{D i} / v_{i}$ $=1.0$, but neglect the electron inhomogeneity by assuming $q_{e}=q_{B}=0$. In the present context this situation is rather artificial, but would correspond to a case in which the ion drift is caused by other mechanisms, such as the $\mathbf{E} \times \mathbf{B}$ drift. Figure 4 shows an unstable peak that is maximum for finite $q_{\|}$ and finite $q_{\perp}$, and also shows the instability for $q_{\perp} \rightarrow 0$, with the same magnitude as that appearing in Fig. 2. Since the electron inhomogeneity has been neglected, these instabilities are caused by the existence of the ion drift, and there-

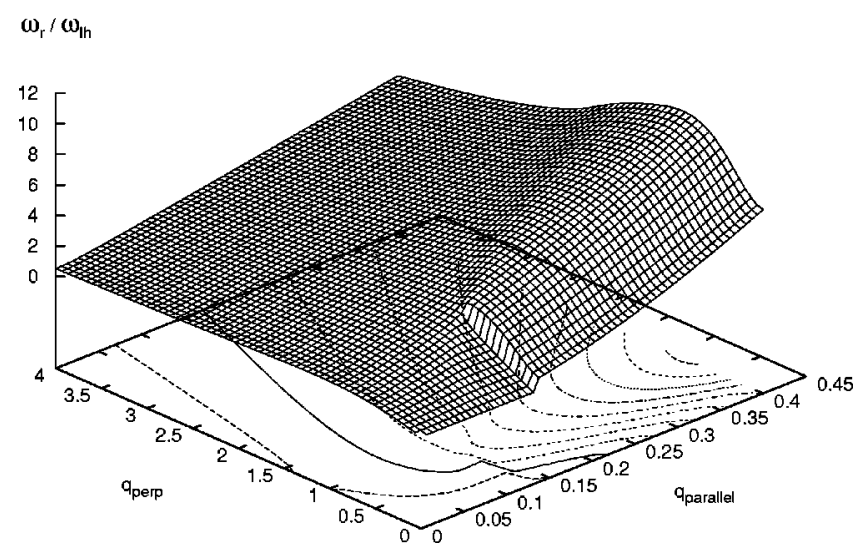

FIG. 3. Dimensionless real frequency $\bar{\omega}_{r}$ as a function of the dimensionless quantities $q_{\|}$and $q_{\perp}$ for $\omega_{p e} /\left|\Omega_{e}\right|=10, v_{D i} / v_{i}$ $=1.0, \beta_{i}=5$, and $\beta_{e}=0$, fully taking into account the electron inhomogeneity. 


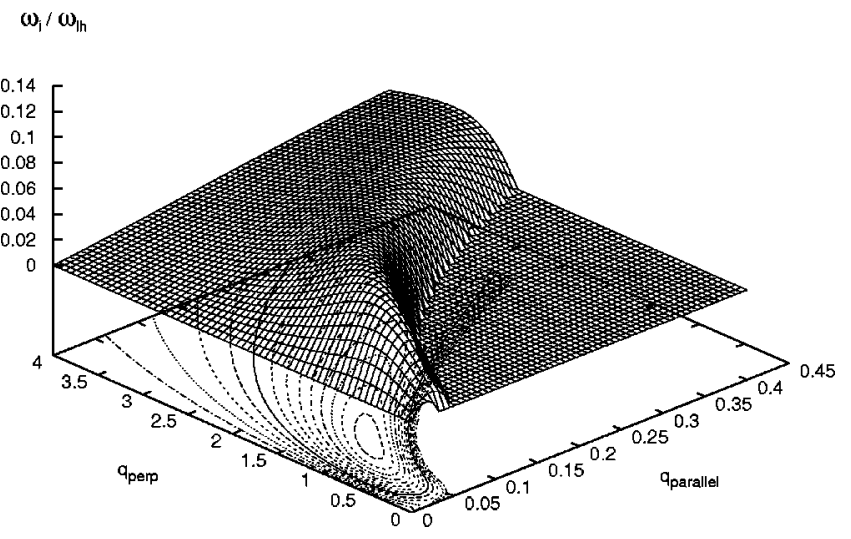

FIG. 4. Dimensionless growth rate $\bar{\omega}_{i}$ as a function of the dimensionless quantities $q_{\|}$and $q_{\perp}$ for $\omega_{p e} /\left|\Omega_{e}\right|=10, v_{D i} / v_{i}=1.0$, $\beta_{i}=5$, and $\beta_{e}=0$, completely neglecting the electron inhomogeneity.

fore are identified as the MTSI and its limit for $q_{\perp} \rightarrow 0$, the IWI $[25,26]$. In Figs. 5 and 6 we show two cases in which the effect of electron inhomogeneity is gradually taken into account, while keeping the same value for the ion drift. Figure 5 displays the growth rates obtained for the case where the values of $q_{e}$ and $q_{B}$ are only $20 \%$ of those utilized in the case of Fig. 2, and Fig. 6 depicts the case with values of $q_{e}$ and $q_{B}$ that are $50 \%$ of those used in Fig. 2. The sequence of figures, Figs. 4, 5, 6, and 2, display the gradual appearance of the LHDI, which occurs near $q_{\|}=0$, due to electron inhomogeneity. Regarding the real frequency, for the cases whose growth rates appear in Figs. 4, 5, 6 we obtained curves almost undistinguishable from that appearing in Fig. 3.

It is interesting to remark that, if the terms containing the space derivatives of the $\chi_{i j}$ are neglected in the dispersion relation, one obtains the MTSI and the IWI, but the LHDI is not found. It is also interesting to remark that, if a WKB approximation is used in Eq. (5) before the introduction of the Gauss law, instead of Eq. (7) we obtain the following familiar set of algebraic equations:

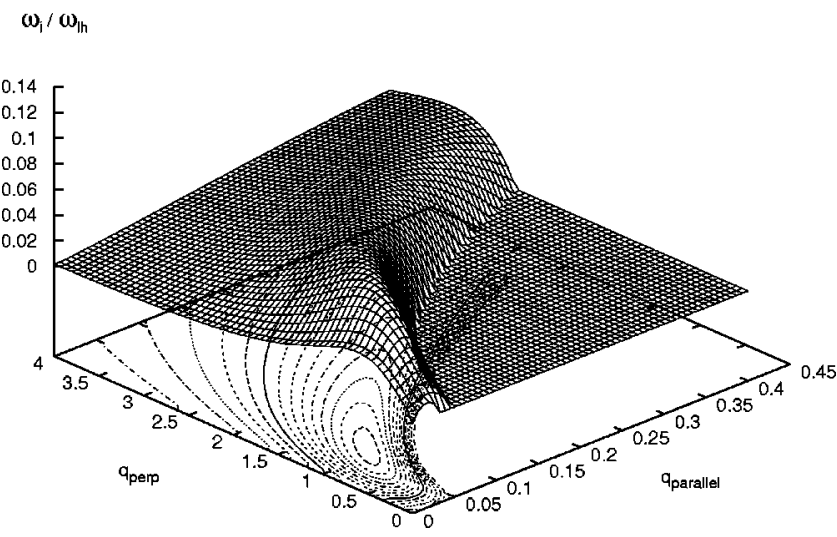

FIG. 5. Dimensionless growth rate $\bar{\omega}_{i}$ as a function of the dimensionless quantities $q_{\|}$and $q_{\perp}$ for $\omega_{p e} /\left|\Omega_{e}\right|=10, v_{D i} / v_{i}=1.0$, $\beta_{i}=5$, and $\beta_{e}=0$, using $q_{e}$ and $q_{B}$ values that are $20 \%$ of the values used in Fig. 2. $\omega_{1} / \omega_{1 h}$

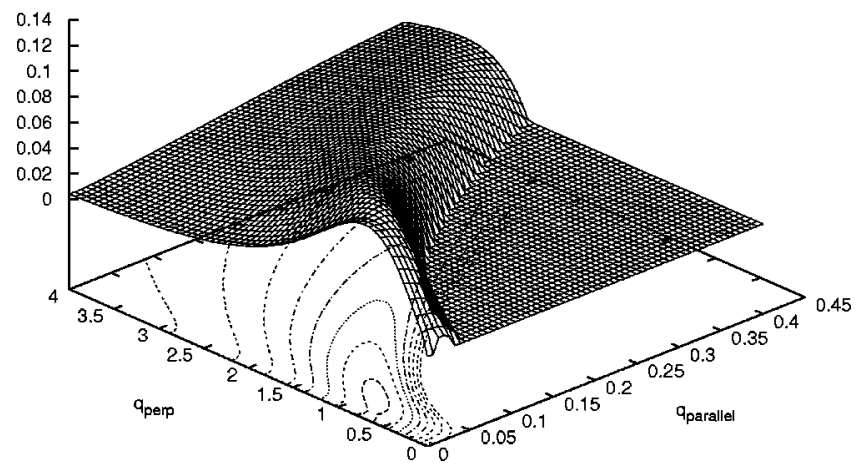

FIG. 6. Dimensionless growth rate $\bar{\omega}_{i}$ as a function of the dimensionless quantities $q_{\|}$and $q_{\perp}$ for $\omega_{p e} /\left|\Omega_{e}\right|=10, v_{D i} / v_{i}=1.0$, $\beta_{i}=5$, and $\beta_{e}=0$, using $q_{e}$ and $q_{B}$ values that are $50 \%$ of the values used in Fig. 2.

$$
\frac{\omega^{2}}{c^{2}} \mathbf{E}+\boldsymbol{k}(\boldsymbol{k} \cdot \mathbf{E})-k^{2} \mathbf{E}+\frac{\omega^{2}}{c^{2}} \overleftrightarrow{\chi} \cdot \mathbf{E}=0,
$$

where the constitutive relation Eq. (8) has been used. The solubility condition for Eq. (16) is the usual dispersion relation

$$
\operatorname{det}\left(k_{i} k_{j}-k^{2} \delta_{i j}+\frac{\omega^{2}}{c^{2}} \varepsilon_{i j}\right)=0
$$

Solving the dispersion relation given by Eq. (17), again we obtain the MTSI and the IWI, with solutions practically equal to those appearing in Fig. 4, but the LHDI is absent. It is seen that the terms with the space derivative of the $\chi_{i j}$ in Eq. (12) are essential for the appearance of the LHDI, and must be maintained in the dispersion relation, since they are of the same order of other inhomogeneity effects that were taken into account in the derivation of the components of the dielectric tensor.

In addition to the argument about the terms with space derivative of the $\chi_{i j}$ contributing with inhomogeneity effects of same order as those taken into account in the evaluation of the $\chi_{i j}$ components themselves, we point out the following. It is well known that, although LHDI is an inherently electromagnetic instability, it has, nevertheless, a strong electrostatic component (see, e.g., discussion in Ref. [13] and references therein). It is also well known that electron inhomogeneities play an important role in the generation of LHDI. Thus, had one considered LHDI electrostatic, as a first approximation, and used Poisson's equation to derive the electrostatic dispersion relation, one would easily obtain LHDI. Moreover, the dispersion relation obtained would be different from the electrostatic limit of Eq. (17). This discrepancy was noted by the authors of Ref. [13], who following the steps of previous authors tried to solve it by using Poisson's equation instead of the $y$ component of Eq. (17), which is based upon Ampère's law. They obtained the LHDI instability, and they have noticed that failure to undertake this remedial procedure would result in a dispersion relation 
that would not describe LHDI at all. Another difficulty faced by the authors of Ref. [13], was that they failed to obtain a unified local description of cross-field instabilities in the lower-hybrid range, having to rely on one dispersion relation in order to obtain LHDI and another for MTSI and IWI. Such a difficulty does not occur in the formulation developed in the present paper, which relies on dispersion relation (15), and which stands as a consistent and unified formulation for the instabilities in the lower-hybrid range of frequencies.

\section{CONCLUSIONS}

As a closing remark, we reiterate that the electron contribution to the components of the dielectric tensor, given in Eqs. (3) and (4), has been obtained following a consistent method that is based on correct description of wave-particle energy exchange [24]. As such, and unlike previous formulations employed in the study of LHDI, the dielectric tensor features correct symmetry properties, which guarantee that the imaginary part of the wave frequency obtained from the dispersion relation is not due to spurious nonresonant terms.

The conventional approaches to describe MTSI have relied on homogeneous theory with the drifts simply imposed (e.g., [8]). In the usual approach to homogeneous theory, the relationship between current density and electric field is utilized in the derivation of the dielectric tensor. For LHDI in high- $\beta$ plasmas, on the other hand, a hybrid formulation that utilize electric current and charge density perturbations has been employed (e.g., $[3,13])$. In the present formulation we have also utilized electric current and charge density fluctuations, which appeared when we took into account Gauss's law of electricity in the derivation of the dispersion relation. Instead of utilizing charge density as an ad hoc procedure, we have shown that the use of Gauss's law introduces a term with a derivative of the space dependent electric conductivity, a term of the same order of other terms responsible for inhomogeneous effects, which have been taken into account in the derivation of the dielectric tensor. This term coming from the use of Gauss's law has been shown to be essential to the proper dispersion relation for inhomogeneous media, and cannot be neglected, otherwise the LHDI is not found as a solution of the dispersion relation in the lower-hybrid range. This would be in contrast with simulation studies, which have reported instabilities that are usually regarded as being the LHDI [27-29], and also in contrast with nonlocal analysis of inhomogeneous equilibria, where unstable solutions identified as the LHDI have been reported many times [30-32].

The conclusion is that the present formalism, derived following a consistent formulation that introduces proper symmetry features, has been shown to accurately describe instabilities in the lower-hybrid range for a weakly inhomogeneous plasma treated in the local approximation, including MTSI, LHDI, and the IWI. It therefore constitutes a unified formulation for the local treatment of the instabilities in the lower-hybrid range, something that was not yet available in the literature.

\section{ACKNOWLEDGMENTS}

This work was partially supported by the Brazilian agencies Conselho Nacional de Desenvolvimento Científico e Tecnológico (CNPq), Fundação de Amparo à Pesquisa do Estado do Rio Grande do Sul (FAPERGS), and Financiadora de Estudos e Projetos (FINEP). P.H.Y. acknowledges National Science Foundation Grant No. ATM 9814197 to the University of Maryland. Discussions with Dr. Ruth S. Schneider are gratefully aknowledged.

\section{APPENDIX A: SOME DETAILS OF THE DERIVATION OF THE DIELECTRIC TENSOR}

According to the previous development of the formalism using the BGI procedure, the electron contribution can be written as follows [14]:

$$
\overleftrightarrow{\chi}^{e}=\overleftrightarrow{\chi}_{P}+\overleftrightarrow{\chi}_{B}
$$

The components $\chi_{P i j}$ may be obtained from Eqs. (27) of Ref. [14]. ${ }^{1}$ For the present case, in which we consider propagation in the $y z$ plane and only terms corresponding to harmonics $n=0$ and $n= \pm 1$ are kept, we obtain the following:

$$
\begin{gathered}
\left(\chi_{P}\right)_{x x}=-\frac{\sqrt{2}}{k_{\|}} \epsilon_{n} X_{e} \nu_{e}^{3} \mathcal{H}_{0}^{\prime}\left(\nu_{e}^{2}\right) Z\left(z_{0}\right), \\
\left(\chi_{P}\right)_{y y}=\frac{1}{\sqrt{2} k_{\|}} \nu_{e} \epsilon_{n} X_{e} \\
\times \sum_{n= \pm 1}\left[\frac{n^{2}}{\nu_{e}^{2}} \mathcal{H}_{n}\left(\nu_{e}^{2}\right)-\frac{1}{Y_{e}} \frac{n}{\nu_{e}^{2}}\right. \\
\left.\times\left(\frac{n^{2}}{\nu_{e}^{2}} \mathcal{H}_{n}\left(\nu_{e}^{2}\right)-\mathcal{H}_{n}^{\prime}\left(\nu_{e}^{2}\right)\right)\right] Z\left(z_{n}\right), \\
\left(\chi_{P}\right)_{z z}=-\frac{1}{2} \frac{k_{\perp}}{k_{\|}^{2}} \epsilon_{n} \frac{X_{e}}{Y_{e}} \mathcal{H}_{0}\left(\nu_{e}^{2}\right) Z^{\prime}\left(z_{0}\right), \\
\left(\chi_{P}\right)_{x y}=-\left(\chi_{P}\right)_{y x}=-\frac{i}{\sqrt{2} k_{\|}} \epsilon_{n} \frac{X_{e}}{Y_{e}} \nu_{e} \mathcal{H}_{0}^{\prime}\left(\nu_{e}^{2}\right) Z\left(z_{0}\right), \\
\left(\chi_{P}\right)_{x z}=-\left(\chi_{P}\right)_{z x}=-\frac{i}{2 k_{\|}} \epsilon_{n} X_{e} \nu_{e}^{2} \mathcal{H}_{0}^{\prime}\left(\nu_{e}^{2}\right) Z^{\prime}\left(z_{0}\right), \\
\left(\chi_{P}\right)_{y z}=\left(\chi_{P}\right)_{z y}=-\frac{1}{4 k_{\|}} \epsilon_{n} \frac{X_{e}}{Y_{e}} \mathcal{H}_{0}^{\prime}\left(\nu_{e}^{2}\right) Z^{(1)}\left(z_{0}\right),
\end{gathered}
$$

where $\nu_{e}^{2}=k_{\perp}^{2} v_{e}^{2} / \Omega_{e}^{2}, \quad z_{0}=\omega /\left(\sqrt{2} k_{\|} v_{e}\right), \quad$ and $\quad z_{n}=(\omega$ $\left.-n \Omega_{e}\right) /\left(\sqrt{2} k_{\|} v_{e}\right)$. Moreover, $X_{e}=\omega_{p e}^{2} / \omega^{2}$ and $Y_{e}=\Omega_{e} / \omega$. According to this definition, the sign that appears in $z_{ \pm}$

\footnotetext{
${ }^{1}$ Notice that there is a typographical error in the second line of Eq. (27b) in Ref. [14]. Instead of “...in] $\mathcal{H}_{n}^{\prime}\left(\nu_{\alpha}^{2}\right)$," the correct expression should be “... \pm in] $\mathcal{H}_{n}^{\prime}\left(\nu_{\alpha}^{2}\right)$."
} 
$=z_{n= \pm 1}$ corresponds to the sign of $n$, and corresponds also to the sign appearing in front of $\left|\Omega_{e}\right|$. Therefore, the sign is opposite to that appearing in front of $\Omega_{e}$, which carries the sign of the charge. Accordingly, the quantity $\nu_{e}$ is a negative quantity, as well as $Y_{e}$.

In the derivation of Eqs. (A2), we have taken into account that, in the vicinity of the lower-hybrid frequency, the absolute value of the arguments of the Fried-Conte functions may be written as follows:

$$
\left|z_{ \pm}\right|=\left|\frac{\omega \mp \Omega_{e}}{\sqrt{2} k_{\|} v_{e}}\right| \simeq\left|\frac{\Omega_{e}}{\sqrt{2} k_{\|} v_{e}}\right|=\frac{\lambda_{\|}}{r_{L e}},
$$

where $r_{L e}$ is the electron Larmor radius and $\lambda_{\|}$represents the parallel wavelength. We have then neglected the contribution of some terms with $n= \pm 1$, as compared to the contribution of those with $n=0$, considering that $\lambda_{\|} \gg r_{L e}$.

The components $\chi_{B i j}$ may be obtained from Ref. [23], where a formulation that keeps relativistic effects has been employed and a more general distribution function has been considered. However, for the present application a nonrelativistic formulation is sufficient. From Eqs. (6) of Ref. [23], by considering only harmonics $n=0$ and $n= \pm 1$, with $\psi$ $=\pi / 2$ (which corresponds in Ref. [23] to propagation in the $y z$ plane), Maxwellian distribution $(l=0)$, and by use of the "nonrelativistic inhomogeneous plasma dispersion function" $\mathcal{G}_{r, p, m, l}$ instead of the "relativistic inhomogeneous plasma dispersion function" $\mathcal{G}_{r, q, p, m, l}$, we arrive at the following:

$$
\begin{gathered}
\left(\chi_{B}\right)_{x x}=-2 \mu_{e} X_{e} \nu_{e}^{2}\left(\mathcal{G}_{0,3,0,0}-\nu_{e}^{2} \mathcal{G}_{0,3,0,1}\right) \\
\left(\chi_{B}\right)_{y y}=-\mu_{e} X_{e} \sum_{n= \pm 1}\left[\mathcal{G}_{0,1,0,1}-2 \nu_{e} \chi_{1 e}\left(\mathcal{G}_{1,2,0,1}-\mathcal{G}_{1,2,1,2}\right)\right] \\
\left(\chi_{B}\right)_{z z}=-\mu_{e} X_{e}\left(\mathcal{G}_{0,1,0,0}+\mu_{e} N_{\|}^{2} \mathcal{G}_{2,1,0,0}\right) \\
\left(\chi_{B}\right)_{x y}=-\left(\chi_{B}\right)_{y x}=i \mu_{e} X_{e} \sum_{n= \pm 1} n \\
\times\left[\mathcal{G}_{0,1,0,1}-\nu_{e}^{2}\left(\mathcal{G}_{0,2,0,1}-\mathcal{G}_{0,2,1,2}\right)\right. \\
\left.+2 \nu_{e} \chi_{1 e}\left(\mathcal{G}_{1,3,1,1}-\nu_{e}^{2} \mathcal{G}_{1,3,1,2}\right)\right] \\
\left(\chi_{B}\right)_{x z}=-\left(\chi_{B}\right)_{z x}=i N_{\|} \mu_{e}^{3 / 2} X_{e} \nu_{e}\left(\mathcal{G}_{1,2,0,0}-v_{e}^{2} \mathcal{G}_{1,2,0,1}\right), \\
\left(\chi_{B}\right)_{y z}=\left(\chi_{B}\right)_{z y}=N_{\|} \mu_{e}^{3 / 2} X_{e} \sum_{n= \pm 1}\left[-n \nu_{e} \mathcal{G}_{1,1,0,1}\right. \\
\left.+n \chi_{1 e}\left(\mathcal{G}_{2,1,0,1}+\mathcal{G}_{2,2,1,1}-\nu_{e}^{2} \mathcal{G}_{2,2,1,2}\right)\right]
\end{gathered}
$$

where $\mu_{e}=m_{e} c^{2} / T_{e}, N_{\|}=c k_{\|} / \omega$, and $\chi_{n e}=n\left(c / v_{e}\right) N_{B} / 2$, where $N_{B}=c \epsilon_{B} / \omega$.

In Eq. (A3), $\mathcal{G}$ is the nonrelativistic inhomogeneous plasma dispersion function for the isotropic case, which may be written as in Eq. (IV-17) of Ref. [21],

$$
\begin{aligned}
\mathcal{G}_{r, p, m, l} & \left(z, \beta, \eta_{\alpha}, \nu_{\alpha}, \chi_{n \alpha}\right) \\
\equiv & -i \int_{0}^{\infty} d t \frac{(i t)^{r} \mathbf{e}^{i z t} \mathbf{e}^{-\beta t^{2}}}{\left(1+i \eta_{\alpha} t\right)^{p}} \\
& \times \mathbf{e}^{-\left(\nu_{\alpha}^{2}+\chi_{n \alpha}^{2} t^{2}\right) /\left(1+i \eta_{\alpha} t\right)} \frac{\left[H_{n \alpha}(t)\right]^{m}}{\left[S_{n \alpha}(t)\right]^{l}} I_{l}\left(\frac{S_{n \alpha}(t)}{1+i \eta_{\alpha} t}\right),
\end{aligned}
$$

where

$$
\begin{gathered}
H_{n \alpha}(t)=\nu_{\alpha}^{2}-i 2 S_{n} \nu_{\alpha} \sin \psi \chi_{n \alpha} t-\chi_{n \alpha}^{2} t^{2}, \\
S_{n \alpha}(t)=\sqrt{\nu_{\alpha}^{4}-2 \nu_{\alpha}^{2} \cos 2 \psi \chi_{n \alpha}^{2} t^{2}+\chi_{n \alpha}^{4} t^{4}} .
\end{gathered}
$$

In the above, $\quad z=\mu_{\alpha}\left(1-n Y_{\alpha}\right)=\mu_{\alpha} \delta_{n \alpha} \quad$ and $\quad \zeta_{\alpha}$ $=N_{B} N_{\perp} \sin \psi / Y_{\alpha}=1-\sigma_{\alpha}$. This nonrelativistic form may be obtained directly from the relativistic inhomogeneous plasma dispersion function, appearing in Ref. [23], by making $i t \rightarrow 0$ and $i \sigma_{\alpha} \rightarrow-i \zeta_{\alpha}$.

Equation (A4) may be further simplified. For instance, in the particular case of $n=0, H_{n e}(t)=\nu_{e}^{2}$ and $S_{n e}(t)=\sqrt{\nu_{e}^{4}}$ $=H_{n e}(t)$, and the inhomogeneous plasma dispersion function for the electron contribution may be written as follows:

$$
\begin{aligned}
\mathcal{G}_{r, p, m, l}\left(z, \beta, \zeta_{e}, \nu_{e}\right) \\
\equiv-i \int_{0}^{\infty} d t \frac{(i t)^{r} \mathbf{e}^{i \mu_{\alpha} \delta_{0 \alpha} t} \mathbf{e}^{-\beta t^{2}}}{\left(1+i \zeta_{e} t\right)^{p}} \\
\quad \times \mathbf{e}^{-\nu_{e}^{2} /\left(1+i \zeta_{e} t\right)} \nu_{e}^{2(m-l)} I_{l}\left(\frac{\nu_{e}^{2}}{1+i \zeta_{e} t}\right) \\
=-i \nu_{e}^{2(m-l)} \int_{0}^{\infty} d t \frac{(i t)^{r} \mathbf{e}^{i \mu_{\alpha} \delta_{0 \alpha} t} \mathbf{e}^{-\beta t^{2}}}{\left(1+i \zeta_{e} t\right)^{p}} \mathcal{H}_{l}\left(\frac{\nu_{e}^{2}}{1+i \zeta_{e} t}\right),
\end{aligned}
$$

where $\zeta_{\alpha}=N_{B} N_{\perp} / Y_{\alpha}$.

For the components depending on harmonics $n= \pm 1$, and weakly inhomogeneous plasmas, such that $\left|\chi_{n e}\right| \ll 1$, we may use the following equation, valid for $n \neq 0$ :

$$
\left.\mathcal{G}_{r, p, m, l} \simeq \mathcal{G}_{r, p, m, l}\right|_{\chi_{n}=0}-\left.\frac{2 m}{\nu_{e}} \sqrt{2 \mu_{e}} N_{\|} S_{n} \chi_{n} \mathcal{G}_{r+1, p, m, l}\right|_{\chi_{n}=0}
$$

where $\chi_{n}=\left(n / 2^{3 / 2}\right) \epsilon_{B} / k_{\|}=\chi_{n e} /\left(\sqrt{2 \mu_{e}} N_{\|}\right)$. For the case of $n= \pm 1$, since $\chi_{n}$ is proportional to $n$, we have $S_{n} \chi_{n}=\chi_{1}$. Equation (A6) shows that we only need the plasma dispersion function evaluated at $\chi_{1}=0$, which means that it is possible to use Eq. (A5) also for $n= \pm 1$, by replacing $\mu_{\alpha} \delta_{n \alpha}$ for $\mu_{\alpha} \delta_{0 \alpha}$, in the argument of the exponential function.

For the particular case of $p=1$, with the use of Eq. (69) of Ref. [33], Eq. (A5) may be written as 


$$
\begin{aligned}
\mathcal{G}_{r, 1, m, l}= & -i 2 \nu_{\alpha}^{2(m-l)} \int_{0}^{\infty} d u u \mathbf{e}^{-u^{2}} J_{l}^{2}\left(\sqrt{2} \nu_{\alpha} u\right) \\
& \times \int_{0}^{\infty} d t(i t)^{r} \mathbf{e}^{i\left(\mu_{\alpha} \delta_{0 \alpha}-\zeta_{\alpha} u^{2}\right) t} \mathbf{e}^{-\beta t^{2}} .
\end{aligned}
$$

We now introduce the definitions of the Fried-Conte function [34],

$$
Z(z)=i \int_{0}^{\infty} d y \exp \left(i z y-y^{2} / 4\right)
$$

Using Eq. (A8), one may express the inhomogeneous plasma dispersion function for $\psi=\pi / 2$ in terms of the derivative of order $r$ of the Fried-Conte function,

$$
\begin{aligned}
\mathcal{G}_{r, 1, m, l}= & -\frac{2 \nu_{\alpha}^{2(m-l)}}{\left(\sqrt{2 \mu_{\alpha}} N_{\|}\right)^{(r+1)}} \int_{0}^{\infty} d u u \mathbf{e}^{-u^{2}} Z^{(r)} \\
& \times\left(\frac{\mu_{\alpha} \delta_{0 \alpha}-\zeta_{\alpha} u^{2}}{\sqrt{2 \mu_{\alpha}} N_{\|}}\right) J_{l}^{2}\left(\sqrt{2} \nu_{\alpha} u\right),
\end{aligned}
$$

where $Z^{(r)}(z)$ represents the derivatives of $Z(z)$ of order $r$.

For the case $p=2$, it is possible to demonstrate a similar expression,

$$
\begin{aligned}
\mathcal{G}_{r, 2, m, l}= & -\frac{\nu_{\alpha}^{2(m-l-1)}}{\left(\sqrt{2 \mu_{\alpha}} N_{\|}\right)^{(r+1)}} \int_{0}^{\infty} d u u \mathbf{e}^{-u^{2}} Z^{(r)} \\
& \times\left(\frac{\mu_{\alpha} \delta_{0 \alpha}-\zeta_{\alpha} u^{2}}{\sqrt{2 \mu_{\alpha}} N_{\|}}\right)\left[\left(2 \nu_{\alpha}^{2} u^{2}-l^{2}\right) J_{l}^{2}\left(\sqrt{2} \nu_{\alpha} u\right)\right. \\
& \left.+2 \nu_{\alpha}^{2} u^{2} J_{l}^{\prime 2}\left(\sqrt{2} \nu_{\alpha} u\right)\right],
\end{aligned}
$$

as well as for $p=3$,

$$
\begin{aligned}
\mathcal{G}_{r, 3, m, l}= & -\frac{\nu_{\alpha}^{2(m-l-2)}}{3\left(\sqrt{2 \mu_{\alpha}} N_{\|}\right)^{(r+1)}} \int_{0}^{\infty} d u u \mathbf{e}^{-u^{2}} Z^{(r)} \\
& \times\left(\frac{\mu_{\alpha} \delta_{0 \alpha}-\zeta_{\alpha} u^{2}}{\sqrt{2 \mu_{\alpha}} N_{\|}}\right)\left\{( 2 \nu _ { \alpha } ^ { 2 } u ^ { 2 } - l ^ { 2 } ) \left[\left(2 \nu_{\alpha}^{2} u^{2}-l^{2}\right)\right.\right. \\
& \left.\times J_{l}^{2}\left(\sqrt{2} \nu_{\alpha} u\right)+2 \nu_{\alpha}^{2} u^{2} J_{l}^{\prime 2}\left(\sqrt{2} \nu_{\alpha} u\right)\right]-l^{2} J_{l}^{2}\left(\sqrt{2} \nu_{\alpha} u\right) \\
& +2 \nu_{\alpha}^{2} u^{2} J_{l}^{\prime 2}\left(\sqrt{2} \nu_{\alpha} u\right) \\
& \left.+2 \sqrt{2} \nu_{\alpha}^{3} u^{3} J_{l}\left(\sqrt{2} \nu_{\alpha} u\right) J_{l}^{\prime}\left(\sqrt{2} \nu_{\alpha} u\right)\right\} .
\end{aligned}
$$

The approximate forms Eqs. (A9), (A10), and (A11) give the nonrelativistic dispersion function for $\chi_{n}=0$, as we need the terms coming from $n= \pm 1$, according to Eq. (A6). Using these explicit expressions for the inhomogeneous plasma dispersion function (A3), after some algebraic manipulations and taking into account that the $Z$ function only depends on $n$ through its argument, we arrive at

$$
\left(\chi_{B}\right)_{x x}=\frac{2 \sqrt{2} \sqrt{\mu_{e}} X_{e}}{N_{\|}} \int_{0}^{\infty} d u u^{3} \mathbf{e}^{-u^{2}} J_{1}^{2}\left(\sqrt{2} \nu_{e} u\right) Z\left(\bar{z}_{0}\right),
$$

$$
\begin{aligned}
\left(\chi_{B}\right)_{y y}= & \frac{\sqrt{2} \sqrt{\mu_{e}} X_{e}}{N_{\|} \nu_{e}^{2}} \\
& \times \sum_{n= \pm 1}\left(\int_{0}^{\infty} d u u \mathbf{e}^{-u^{2}} Z\left(\bar{z}_{n}\right) J_{1}^{2}\left(\sqrt{2} \nu_{e} u\right)\right. \\
& -\chi_{1} 2 \sqrt{2} Z^{\prime}\left(z_{n}\right) \int_{0}^{\infty} d u u^{2} \mathbf{e}^{-u^{2}} \\
& \left.\times J_{1}\left(\sqrt{2} \nu_{e} u\right) J_{2}\left(\sqrt{2} \nu_{e} u\right)\right)
\end{aligned}
$$$$
\left(\chi_{B}\right)_{z z}=-\frac{X_{e}}{N_{\|}^{2}} \int_{0}^{\infty} d u u\left(\mu_{e} \delta_{0 e}\right.
$$$$
\left.-\zeta_{e} u^{2}\right) \mathbf{e}^{-u^{2}} J_{0}^{2}\left(\sqrt{2} \nu_{e} u\right) Z^{\prime}\left(\bar{z}_{0}\right),
$$

$$
\begin{aligned}
& \left(\chi_{B}\right)_{x y}=-\left(\chi_{B}\right)_{y x} \\
& =i \frac{2 \sqrt{\mu_{e}} X_{e}}{N_{\|} \nu_{e}} \sum_{n= \pm 1} n \\
& \times\left(\int_{0}^{\infty} d u u^{2} \mathbf{e}^{-u^{2}} Z\left(\bar{z}_{n}\right)\right. \\
& \times J_{1}\left(\sqrt{2} \nu_{e} u\right) J_{1}^{\prime}\left(\sqrt{2} \nu_{e} u\right) \\
& \left.+\sqrt{2} \chi_{1} Z^{\prime}\left(z_{n}\right) \int_{0}^{\infty} d u u^{3} \mathbf{e}^{-u^{2}} J_{2}^{2}\left(\sqrt{2} \nu_{e} u\right)\right), \\
& \left(\chi_{B}\right)_{x z}=-\left(\chi_{B}\right)_{z x}=i \frac{\sqrt{2} \sqrt{\mu_{e}} X_{e}}{N_{\|}} \int_{0}^{\infty} d u u^{2} \mathbf{e}^{-u^{2}} \\
& \times J_{0}\left(\sqrt{2} \nu_{e} u\right) J_{1}\left(\sqrt{2} \nu_{e} u\right) Z^{\prime}\left(\bar{z}_{0}\right), \\
& \left(\chi_{B}\right)_{y z}=\left(\chi_{B}\right)_{z y}=\frac{\sqrt{\mu_{e}} X_{e} \nu_{e}^{-2}}{N_{\|}} \\
& \times \sum_{n= \pm 1}\left(n \nu_{e} \int_{0}^{\infty} d u u \mathbf{e}^{-u^{2}} Z^{\prime}\left(\bar{z}_{n}\right) J_{1}^{2}\left(\sqrt{2} \nu_{e} u\right)\right. \\
& +2 \chi_{1} n\left[z_{n} Z^{\prime}\left(z_{n}\right)\right. \\
& \left.+Z\left(z_{n}\right)\right] \int_{0}^{\infty} d u u \mathbf{e}^{-u^{2}}\left[J_{1}^{2}\left(\sqrt{2} \nu_{e} u\right)\right. \\
& \left.\left.+\sqrt{2} \nu_{e} u J_{1}\left(\sqrt{2} \nu_{e} u\right) J_{2}\left(\sqrt{2} \nu_{e} u\right)\right]\right),
\end{aligned}
$$

where $\bar{z}_{n}=\left(\mu_{e} \delta_{n e}-\zeta_{e} u^{2}\right) /\left(\sqrt{2 \mu_{e}} N_{\|}\right)=\left(\omega-n \Omega_{e}-k_{\perp} \epsilon_{B} v_{e}^{2} u^{2} /\right.$ $\left.\Omega_{e}\right) /\left(\sqrt{2} k_{\|} v_{e}\right)$. As mentioned in Sec. II, the ions can be considered unmagnetized and carry a current in the $y$ direction. Their contribution to the dielectric tensor may be given by Eq. (4), which is the same as that which appears in Ref. [13]. 
Up to this point, we have employed the same notation used in previous works, in order to relate more directly the expressions useful for the present application to those previously obtained $[14,15,22,23]$. In what follows, however, it may be more convenient to modify the notation and write the dielectric tensor in terms of quantities more directly connected to the lower-hybrid range. These are the normalized quantities that we have already encountered in Eqs. (3) and (4): $q_{\perp}=c k_{\perp} / \omega_{p e}, \quad q_{\|}=c k_{\|} / \omega_{p e}, \quad q_{n}=\left(c / \omega_{p e}\right) \epsilon_{n}$, $q_{B}=\left(c / \omega_{p e}\right) \epsilon_{B}$, and $\bar{\omega}=\omega / \omega_{l h}$.

It may be useful to remark here that the definitions of $v_{e}$, $v_{i}$, and $v_{D i}$ used in the present work are different from those used in Ref. [13], while the definition of $v_{A}$ is the same appearing in that paper. The nondimensional quantity $q_{n}$ can be related to the ion drift velocity [13], $q_{n}$ $=2\left(v_{D i} / v_{i}\right) / \sqrt{\beta_{i}\left(m_{i} / m_{e}\right)}$, with the relationship between $q_{n}$ and $q_{B}, q_{B}=\left(\beta_{e}+\beta_{i}\right) q_{n} / 2$, where $\beta_{\alpha}=8 \pi n_{0} T_{\alpha} / B_{0}^{2}$. Using this more convenient notation, the components of $\overleftrightarrow{\chi}^{e}$ and $\overleftrightarrow{\chi}^{i}$ may be written as they appear in Eqs. (3) and (4), in the main body of the paper.

\section{APPENDIX B: EVALUATION OF THE SPACE DERIVATIVE OF THE DIELECTRIC TENSOR}

In order to evaluate the $x$ derivative of the $\chi_{i j}$ components, let us start by considering that, when the magnetic field inhomogeneity is taken into account in the evaluation of characteristic trajectories, and the distribution function is expanded up to first order in the inhomogeneity, the susceptibility tensor that takes into account the inhomogeneity effects may be written as a summation of a term incorporating effects of field gradients and depending on the distribution function, and another term depending on the derivative of the distribution function,

$$
\overleftrightarrow{\chi}^{0} \simeq \overleftrightarrow{\chi}_{1}^{0}\left[f_{e}\left(\epsilon_{n}=0\right), \epsilon_{B}\right]+\overleftrightarrow{\chi}_{2}^{0}\left[f_{e}^{\prime}, \epsilon_{B}=0\right]
$$

The first term in Eq. (B1), which incorporates effects of the field inhomogeneity and neglects the density inhomogeneity, is obtained from the derivation made in Ref. [22],

$$
\begin{aligned}
\overleftrightarrow{\chi}_{B}^{0}= & -i \frac{4 \pi}{\omega} \frac{e^{2}}{m_{e}} \sum_{n} \int d^{3} p \int d \tau p_{\perp} \mathcal{L}\left(f_{e 0}\right) \\
& \times \vec{\Sigma}_{n e}^{*} \vec{\Pi}_{n e} e^{-n \theta_{n e}(\tau)} e^{i D_{n e} \tau}-\hat{\mathbf{e}}_{3} \hat{\mathbf{e}}_{3} \frac{4 \pi}{\omega} \frac{e^{2}}{m_{e}} \int d^{3} p \frac{p_{\|}}{\gamma} L\left(f_{e 0}\right),
\end{aligned}
$$

where the space dependence appears through the quantity

$$
D_{n e}\left(\epsilon_{B}\right)=\gamma \omega-c k_{\|} u_{\|}-n \Omega_{e}\left(1+\epsilon_{B} x\right)-\epsilon_{B} \frac{k_{\perp} u_{\perp}^{2} c^{2}}{2 \Omega_{e}} \sin \psi .
$$

Explicit expressions for other quantities involved can be found in Ref. [22].
The second contribution to Eq. (B1), which is of order $\epsilon_{n}$, will not be important in what follows. It can be obtained according to the derivation found in Ref. [15].

Evaluating the derivative of $\hat{\chi}_{i j}^{0}$, using Eq. (B1), we get

$$
\begin{aligned}
\partial_{x} \chi_{i j}^{0} \simeq & i \epsilon_{B} \frac{4 \pi}{\omega} \frac{e^{2}}{m_{e}} \sum_{n} n \Omega_{e} \int d^{3} p \int d \tau(i \tau) p_{\perp} \mathcal{L}\left(f_{e 0}\right) \\
& \times\left(\Sigma_{n e}^{*}\right)_{i}\left(\Pi_{n e}\right)_{j} e^{-n \theta_{n e}(\tau)} e^{i D_{n e} \tau} .
\end{aligned}
$$

Applying the BGI transformation, following what has been made for the components $\chi_{i j}$ themselves in Ref. [22], the outcome is the following:

$$
\begin{aligned}
\partial_{x} \chi_{i j}= & i \epsilon_{B} \frac{4 \pi e^{2}}{m_{e} \omega} \sum_{n} n \Omega_{e} \int d^{3} p \int d \tau(i \tau) p_{\perp} \mathcal{L}\left(f_{e 0}\right) \\
& \times\left(\Pi_{n e}^{-}\right)_{i}\left(\Pi_{n e}^{+}\right)_{j} e^{-n \theta_{n e}(\tau)} e^{i D_{n e} \tau} .
\end{aligned}
$$

Each term in this summation is equivalent to the terms appearing in the $\chi_{B i j}^{0}$, multiplied by $-n \Omega_{e} \epsilon_{B}(i \tau)$. When taking into account the distribution function, in the nonrelativistic approximation, the velocity integrals will be the same as those appearing in the evaluation of the $\chi_{i j}$, with the $\tau$ integral corresponding to the $\mathcal{G}_{r+1, p, m, l}^{n r}$ instead of the $\mathcal{G}_{r, p, m, l}^{n r}$. Proceeding similarly to what we did to obtain Eqs. (A3), we obtain

$$
\begin{gathered}
\partial_{x} \chi_{11}=0 \\
\partial_{x} \chi_{22}=\epsilon_{B} \mu_{e}^{2} X_{e} Y_{e} \sum_{n= \pm 1} n\left[\mathcal{G}_{1,1,0,1}-2 \nu_{e} \chi_{1 e}\left(\mathcal{G}_{2,2,0,1}\right.\right. \\
\left.\left.-\mathcal{G}_{2,2,1,2}\right)\right], \\
\partial_{x} \chi_{B 33}=0, \\
\partial_{x} \chi_{B}\left({ }_{21}^{12}\right)= \pm \epsilon_{B} i \mu_{e}^{2} X_{e} Y_{e} \\
\times \sum_{n= \pm 1} n^{2}\left[\mathcal{G}_{1,1,0,1}-\nu_{e}^{2}\left(\mathcal{G}_{1,2,0,1}-\mathcal{G}_{1,2,1,2}\right)\right. \\
\left.+2 \nu_{e} \chi_{1 e}\left(\mathcal{G}_{2,3,1,1}-\nu_{e}^{2} \mathcal{G}_{2,3,1,2}\right)\right], \\
\partial_{x} \chi_{B}\left(\begin{array}{l}
13 \\
31
\end{array}\right)=-0,
\end{gathered}
$$$$
\partial_{x} \chi_{B}\left(\begin{array}{l}
23 \\
32
\end{array}\right)=-\epsilon_{B} N_{\|} \mu_{e}^{5 / 2} X_{e} Y_{e}
$$$$
\times \sum_{n= \pm 1} n^{2}\left[-\nu_{e} \mathcal{G}_{2,1,0,1}+\chi_{1 e}\right.
$$$$
\left.\times\left(\mathcal{G}_{3,1,0,1}+\mathcal{G}_{3,2,1,1}-\nu_{e}^{2} \mathcal{G}_{3,2,1,2}\right)\right] .
$$

For the evaluation of these expressions, we use Eqs. (A4)-(A11), as we did in Appendix A, and obtain 


$$
\partial_{x} \chi_{i j}=-\epsilon_{B} \eta_{i j}
$$

where

$$
\begin{gathered}
\eta_{x x}=0, \\
\eta_{22}=\frac{\mu_{e} X_{e} Y_{e}}{N_{\|}^{2} \nu_{e}^{2}} \sum_{n= \pm 1} n \int_{0}^{\infty} d u u \mathbf{e}^{-u^{2}} \\
\times Z^{(1)}\left(\frac{\mu_{e} \delta_{n e}-\zeta_{e} u^{2}}{\sqrt{2 \mu_{e}} N_{\|}}\right) J_{1}^{2}\left(\sqrt{2} \nu_{e} u\right), \\
\eta_{33}=0,
\end{gathered}
$$

$$
\begin{aligned}
\eta\left(\begin{array}{l}
12 \\
21
\end{array}\right)= & \pm i \frac{\sqrt{2} \mu_{e} X_{e} Y_{e}}{N_{\|}^{2} \nu_{e}} \sum_{n= \pm 1} n^{2} \int_{0}^{\infty} d u u^{2} \mathbf{e}^{-u^{2}} \\
& \times Z^{(1)}\left(\frac{\mu_{e} \delta_{n e}-\zeta_{e} u^{2}}{\sqrt{2 \mu_{e}} N_{\|}}\right) J_{1}\left(\sqrt{2} \nu_{e} u\right) \\
& \times J_{1}^{\prime}\left(\sqrt{2} \nu_{e} u\right), \\
\eta\left(\begin{array}{l}
23 \\
32
\end{array}\right)= & \frac{\mu_{e} X_{e} Y_{e}}{\sqrt{2} N_{\|}^{2} \nu_{e}} \sum_{n= \pm 1} n^{2} \int_{0}^{\infty} d u u \mathbf{e}^{-u^{2}} \\
& \times Z^{(2)}\left(\frac{\mu_{e} \delta_{n e}-\zeta_{e} u^{2}}{\sqrt{2 \mu_{e}} N_{\|}}\right) J_{1}^{2}\left(\sqrt{2} \nu_{e} u\right) .
\end{aligned}
$$

[1] N.A. Krall and P.C. Liewer, Phys. Rev. A 4, 2094 (1971).

[2] R.C. Davidson and N.T. Gladd, Phys. Fluids 18, 1327 (1975).

[3] N.T. Gladd, Phys. Fluids 18, 1327 (1975).

[4] R.C. Davidson, N.T. Gladd, C.S. Wu, and J.D. Huba, Phys. Fluids 20, 301 (1977).

[5] J.B. Hsia, S.M. Chu, M.F. Hsia, R.L. Chou, and C.S. Wu, Phys. Fluids 22, 1737 (1979).

[6] J.D. Huba, N.T. Gladd, and J.F. Drake, J. Geophys. Res. A 86, 5881 (1981).

[7] Y.M. Zhou, H.K. Wong, C.S. Wu, and D. Winske, J. Geophys. Res. A 88, 3026 (1983).

[8] J.B. McBride, E. Ott, J.P. Boris, and J.H. Orens, Phys. Fluids 15, 2367 (1972).

[9] D.S. Lemons and S.P. Gary, J. Geophys. Res. A 83, 1625 (1978).

[10] C.S. Wu, Y.M. Zhou, S.T. Tsai, S.C. Guo, D. Winske, and K. Papadopoulos, Phys. Fluids 26, 1259 (1983).

[11] S.T. Tsai, M. Tanaka, J.D. Gaffey, E.H. da Jornada, C.S. Wu, and L.F. Ziebell, J. Plasma Phys. 32, 159 (1984).

[12] A.T.Y. Lui, C.-L. Chang, A. Mankofsky, H.-K. Wong, and D. Winske, J. Geophys. Res. A 96, 11389 (1991).

[13] P.H. Yoon, A.T.Y. Lui, and C.-L. Chang, Phys. Plasmas 1, 3033 (1994).

[14] R. Gaelzer, L.F. Ziebell, and O.J.G. Silveira, Phys. Plasmas 6, 4533 (1999).

[15] R.A. Caldela Filho, R.S. Schneider, and L.F. Ziebell, J. Plasma Phys. 42, 165 (1989).

[16] R. A. Caldela Filho, Ph.D. thesis, Universidade Federal do Rio Grande do Sul, 1990.
[17] R.A. Caldela Filho, R.S. Schneider, and L.F. Ziebell, J. Plasma Phys. 43, 335 (1990).

[18] C.J. de H. Cavalcanti, M.Sc. dissertation, Universidade Federal do Rio Grande do Sul, 1994.

[19] C.J.H. Cavalcanti, R.S. Schneider, and L.F. Ziebell, J. Plasma Phys. 52, 195 (1994).

[20] R.S. Schneider, L.F. Ziebell, and R. Gaelzer, Phys. Plasmas 4, 3091 (1997).

[21] R. Gaelzer, Ph.D. thesis, Universidade Federal do Rio Grande do Sul, 1995.

[22] R. Gaelzer, R.S. Schneider, and L.F. Ziebell, Phys. Rev. E 51, 2407 (1995).

[23] R. Gaelzer, R.S. Schneider, and L.F. Ziebell, Phys. Rev. E 55, 5859 (1997).

[24] V.S. Beskin, A.V. Gurevich, and Y.I. Istomin, Zh. Eksp. Teor. Fiz. 92, 1277 (1987) [Sov. Phys. JETP 65, 715 (1987)]. .

[25] C.L. Chang, H.K. Wong, and C.S. Wu, Phys. Rev. Lett. 65, 1104 (1990).

[26] P.H. Yoon and A.T.Y. Lui, Phys. Fluids B 5, 836 (1993).

[27] D. Winske, Phys. Fluids 24, 1069 (1981).

[28] M. Tanaka and T. Sato, J. Geophys. Res. A 86, 5541 (1981).

[29] J.U. Brackbill, D.W. Forslund, K.B. Quest, and D. Winske, Phys. Fluids 27, 2682 (1984).

[30] D.B. Batchelor and R.C. Davidson, Phys. Fluids 19, 882 (1976).

[31] R.C. Davidson, Phys. Fluids 19, 1189 (1976).

[32] J.D. Huba, J.F. Drake, and N.T. Gladd, Phys. Fluids 23, 552 (1980).

[33] P.A. Robinson, J. Math. Phys. 27, 1206 (1986).

[34] B.D. Fried and S.D. Conte, The Plasma Dispersion Function (Academic Press, New York, 1961). 